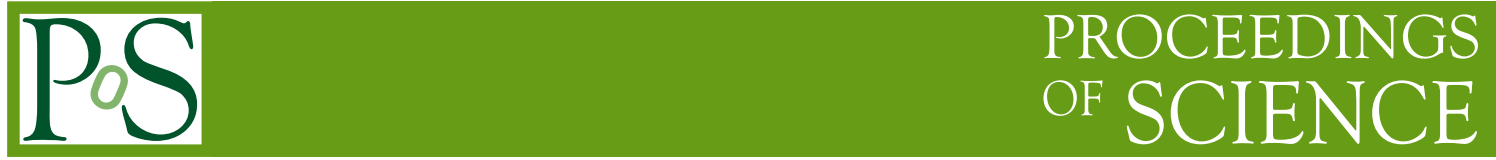

\title{
Saturation, coherence and exclusive final states
}

\section{Krzysztof Kutak*}

Instytut Fizyki Jądrowej im. H. Niewodniczańskiego

Radzikowskiego 152, 31-342 Krakow, Poland

E-mail: krzysztof.kutak@ifj.edu.pl

\section{Dawid Toton}

Instytut Fizyki Ja̧drowej im. H. Niewodniczańskiego

Radzikowskiego 152, 31-342 Krakow, Poland

E-mail: dawid.toton@ifj.edu.pl

We report of recently obtained solution of low $x$ limit of KGBJS equation. The solution of the KGBJS equation is compared to gluon densities resulting from the CCFM. As the saturation scale $Q_{s}$ now becomes available as a function of the hard scale $p$ we observe that low values of $p$ impede its growth with $\frac{1}{x}$. Also, at values much larger than partons transversal momentum the saturation effects become independent on the hard scale what we call liberation of saturation scale.

XXI International Workshop on Deep-Inelastic Scattering and Related Subject-DIS2013, 22-26 April 2013

Marseilles,France

\footnotetext{
* Speaker.
} 


\section{Introduction}

Quantum Chromodynamics (QCD) is a theory which is used to set up the initial conditions for the collisions at the LHC as well as to calculate properties of hadronic observables. In order to apply perturbative QCD to scattring process one decomposes the scross section into long distance part, called parton density, and hard matrix element. In particular here we will focus on high energy factorization $[1,2]$. The evolution equations of high energy factorization resum logarithms of energy accompanied by a strong coupling constant, i.e. terms proportional to $\alpha_{s}^{n} \ln ^{m} s / s_{0}$. This framework applies if the total energy of a scattering process is much bigger than any other hard scale involved in a scattering process.

With the LHC one entered into a region of phase space where both the energy and momentum transfers are high and partons form a dense system allowing in principle for parton saturation [1]. Recently a framework has been provided in $[3,4,5]$ where both dense systems and hard processes at high energies can be studied (for another approach to this problem we refer the Reader to [6]). In the following we report on analysis of equation proposed in [3] and extraction of hard scale dependent saturation scale as performed in [7].

\section{The KGBJS evolution equation}

\subsection{Hard emissions approximation and running coupling effects}

The KGBJS equation in low $\mathrm{x}$ limit reads ${ }^{1}$ :

$$
\begin{aligned}
\mathscr{E}(x, k, p) & =\mathscr{E}_{0}(x, k, p) \\
& +\int_{x}^{x_{0}} \frac{\mathrm{d} w}{w} \int_{0}^{\infty} \frac{\mathrm{d} q^{2}}{q^{2}} \int_{0}^{\pi} \frac{\mathrm{d} \phi}{\pi} \theta(p-z q) P_{g g}(z, k, q) \mathscr{E}\left(w, k^{\prime}, q\right) \\
& -\frac{1}{\pi R^{2}} \int_{x}^{x_{0}} \frac{\mathrm{d} w}{w} \theta(p-z k) P_{g g}(z, k, k) \mathscr{E}^{2}(w, k, k)
\end{aligned}
$$

The momentum vector associated with $i$-th emitted gluon is

$$
q_{i}=\alpha_{i} p_{P}+\beta_{i} p_{e}+q_{t i}
$$

The variable $p$ in (2.1) is defined via $\bar{\xi}=p^{2} /\left(x^{2} s\right)$ where $\frac{1}{2} \ln (\bar{\xi})$ is a maximal rapidity which is determined by the kinematics of hard scattering, $\sqrt{s}$ is the total energy of the collision and $k^{\prime}=|\boldsymbol{k}+\boldsymbol{q}|, \bar{\alpha}=N_{c} \alpha_{s} / \pi$. We also define $k \equiv|\mathbf{k}|$. The momentum $\mathbf{q}$ is the transverse momentum of the emitted gluon. The form factor $\Delta_{n s}$ accompanying the $1 / z$ pole accounts for angular ordering. We use its form as proposed in [9]:

$$
\Delta_{n s}(z, k, q)=\exp \left(-\bar{\alpha}_{s} \ln \frac{z_{0}}{z} \ln \frac{k^{2}}{z_{0} z q^{2}}\right)
$$

\footnotetext{
${ }^{1}$ In the present paper we are going to solve the equation in the approximate form where the eventual problem observed in [8] does not show up. This problem will be addressed in the future.
} 


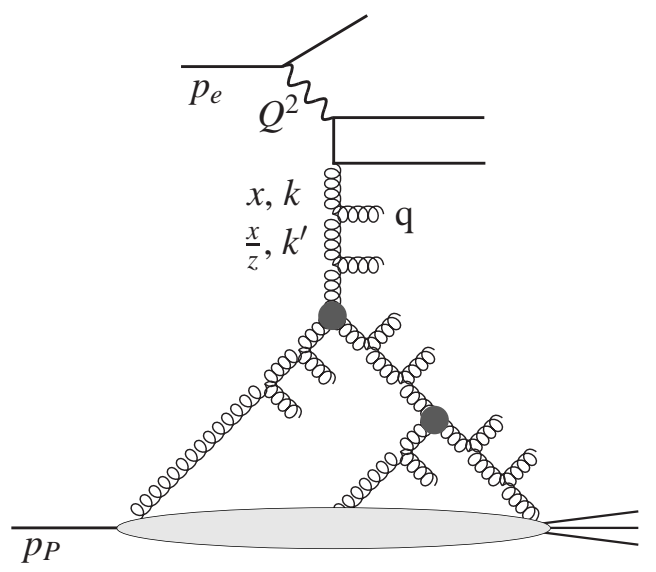

Figure 1: Schematic illustration of kinematical variables used in the KGBJS equation

where $z_{0}=\frac{k}{q}$ for $z<\frac{k}{q}<1$ and outside the interval it assumes the bounding values, $z_{0}=z$ when $\frac{k}{q}<z$ and $z_{0}=1$ when $\frac{k}{q}>1$. where $z=\frac{x}{w}$ under both of the $\mathrm{d} w$ integrals (from now on when we will use the KGBJS acronym we will refer to equation (2.1). The splitting function, with running $\alpha_{s}$ following [9], is simplified to:

$$
P_{g g}(z, k, q)=\bar{\alpha}_{s}\left(k^{2}\right) \frac{\Delta_{n s}(z, k, q)}{z} .
$$

The parameter characterizing the target is chosen to be $R=10 / \sqrt{\pi}$ and the starting point of evolution is chosen to be $x_{0}=10^{-2}$. The running coupling corrections were included via the one loop formula. The initial condition we choose to be:

$$
\mathscr{E}_{0}(x, k, p)=\frac{\mathrm{GeV}}{k} \mathrm{e}^{\bar{\alpha}_{s}\left(k^{2}\right) \ln \frac{x_{0}}{x} \ln \frac{k^{2}}{\mu^{2}}} .
$$

The extra $x$-dependent term is motivated by the resummation procedure, its role is to attenuate the gluon density with decreasing $x$. As we see on Fig. (2) showing the $x$ dependence of solutions at small $p$ considered form of the initial condition leads to falling distribution of the CCFM and KGBJS equations. This is not the case for the BK equation as we observed in [7]. The particularly interesting is the behavior of the CCFM and the KGBJS aequations as a function of hard scale related variable $p$. The Fig. (3) shows that the solution of the equations is a constant function of the $p$ variable as it is larger than transversal momentum of gluon. This effect can be understood by investigating the $\theta(p-z q)$ function in the considered equations. If the variable $p$ is larger than $k$ then the theta function sets to one and the angular ordering is relaxed. This has interesting implications for the saturation scale generated by the KGBJS equation. The plots on Fig. (2) compare solutions of CCFM and KGBJS. We see the damping of the gluon density due to nonlinearity in case of KGBJS equation as we go towards low $x$ and low $k$ values. 


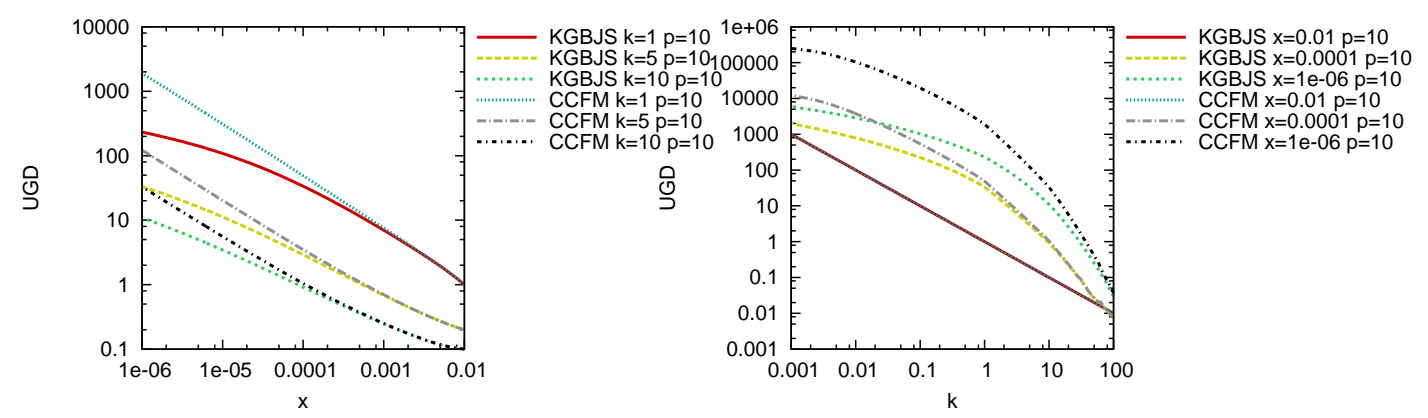

Figure 2: Comparison of solutions of the KGBJS and CCFM equations.

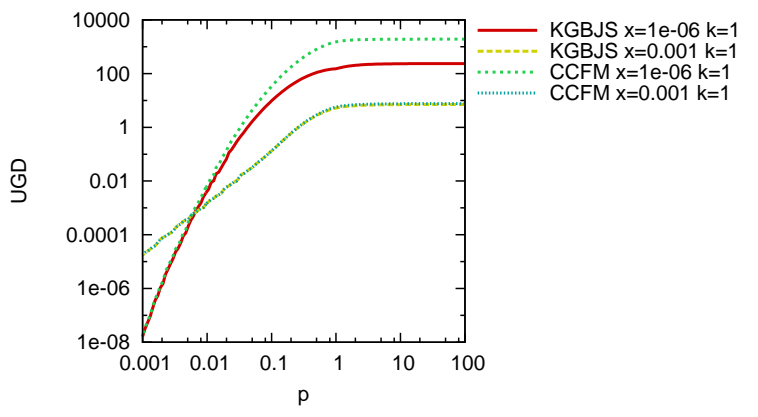

Figure 3: Hard scale dependence of the CCFM and KGBJS equations.

\section{Saturation of the exclusive gluon distribution}

To shed light on the importance of nonlinear corrections in the KGBJS, we consider contour lines of the relative difference between solutions:

$$
\beta(x, k, p)=\frac{\left|\mathscr{E}_{C C F M}(x, k, p)-\mathscr{E}_{K G B J S}(x, k, p)\right|}{\mathscr{E}_{C C F M}(x, k, p)} .
$$

The traditional saturation scale $Q_{s}$, i.e. transversal momentum for which the effects of nonlinearity are noticeable, we define as:

$$
\beta\left(x, Q_{s}(x, p), p\right)=\text { const } .
$$

The quantity defined above, as observed in [10], has somewhat different slope compared to the saturation scale defined as a scale where the dipole amplitude is $1 / 2$. However, as we see from the plots it is a good measure of the strength of nonlinearities. The plot of $\beta$ on Fig. (4) confirms the familiar growth of the saturation scale, which can be seen as $1 / x$ is increasing upwards on the plot.

The most interesting and novel effect as compared to previously known results is the dependence of the saturation scale on the hard scale related variable $p$. Several cross-sections of the $\beta$ function (we limit ourselves to the running coupling case since the fixed coupling case does not bring anything new) on Fig. (4) indicate regions where KGBJS solutions diverge from results of 

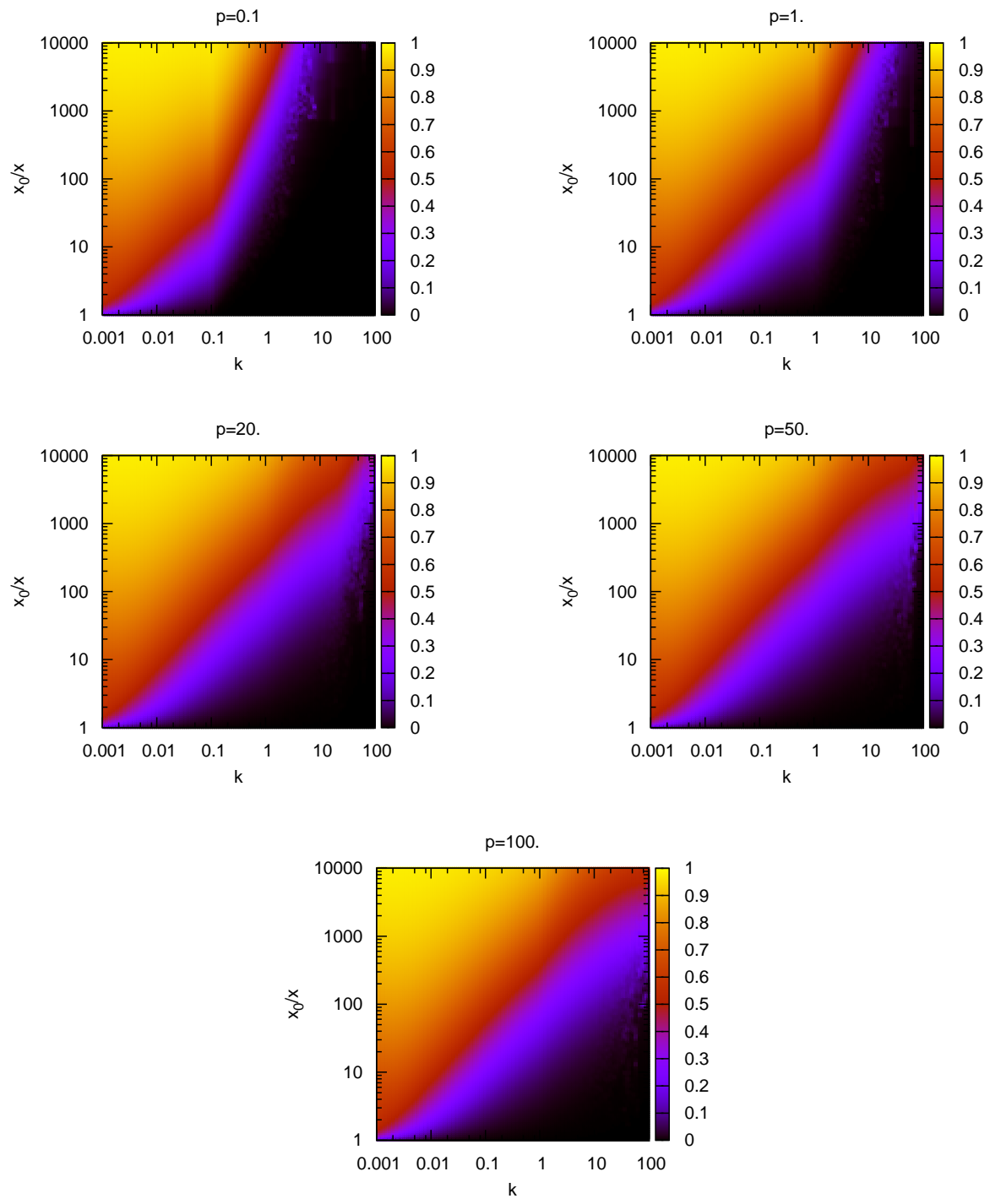

Figure 4: The $\beta$ function (cross-sections for constant $p$ ). Solutions with running $\alpha_{s}$.

the linear evolution. The $k>p$ areas of the plots show that the nonlinear effects enter when the $x_{0} / x$ is rather small. We also see that at $p \approx k$ the saturation line changes slope to larger value and as we go towards larger $k$ the saturation is weaker. Similar effect has been already observed in [11]. The difference is however in the strength of the effect since in the absorptive boundary method the authors of [11] set arbitrarily the value of gluon density below the saturation scale to a constant value while in our approach we allow for dynamical evolution and growth of gluon density. We also see that with growing $p$ the nonlinear effects become larger, the slope becomes approximately constant and gluons get blocked by saturation. This is the consequence of larger available phase space (note the $\theta(p-z \bar{q})$ factor in the kernel of the Eq. 2.1) for larger $p$ which allows for the gluon density to grow and therefore to come at values where the nonlinear effects start to be important. Eventually in phase space region where $p \gg k$ the KGBJS equation becomes independent on the 
hard scale and therefore the saturation scale stops to depend on it. The effect, called here liberation of saturation scale, is linked to the so-called saturation of saturation scale expected in $[3,11]$. Since as we go towards the smaller values of $p$ we see that the saturation bends towards the $x_{0} / x$ axis and its growth is hindered.

\section{Conclusions}

In this paper we reported on recent numerical study of the simplified form of the KGBJS and CCFM evolution equations with running coupling constant. We investigated the role of nonlinearity in the KGBJS equation by studying the emergent saturation scale i.e. the relative differences between solutions of the KGBJS and CCFM equations. Due to the dependence of the KGBJS equation on the hard scale the saturation scale has been shown to depend on it in a nontrivial way. In particular, when the hard scale gets much larger than the $k$ of the gluon, the saturation scale stops to depend on hard scale value and liberates itself to become a function of $x$ and $k$ only.

\section{Acknowledgments}

Krzysztof Kutak would like to thank the organizers for invitation to give a talk at DIS 2013. This research has been supported by Narodowe Centrum Badań i rozwoju with grant LIDER/02/35/L-2/10/NCBiR/2011.

\section{References}

[1] L. V. Gribov, E. M. Levin and M. G. Ryskin, Phys. Rept. 100 (1983) 1.

[2] S. Catani, M. Ciafaloni and F. Hautmann, Nucl. Phys. B 366 (1991) 135.

[3] K. Kutak, K. Golec-Biernat, S. Jadach and M. Skrzypek, JHEP 1202 (2012) 117.

[4] K. Kutak, arXiv:1206.1223 [hep-ph].

[5] K. Kutak, JHEP 1212 (2012) 033 [arXiv:1206.5757 [hep-ph]].

[6] C. Flensburg, G. Gustafson and L. Lonnblad, JHEP 1108 (2011) 103 [arXiv:1103.4321 [hep-ph]].

[7] K. Kutak and D. Toton, arXiv:1306.3369 [hep-ph].

[8] M. Deak, arXiv:1209.6092 [hep-ph].

[9] J. Kwiecinski, A. D. Martin and P. J. Sutton, Phys. Rev. D 52 (1995) 1445 [hep-ph/9503266].

[10] K. Kutak and A. M. Stasto, Eur. Phys. J. C 41 (2005) 343 [hep-ph/0408117].

[11] E. Avsar and A. M. Stasto, JHEP 1006 (2010) 112 [arXiv:1005.5153 [hep-ph]]. 